\title{
Standardization of CT Depiction of Cochlear Implant Insertion Depth
}

\author{
C.C. Colby, N.W. Todd, H.R. Harnsberger, and P.A. Hudgins
}

\begin{abstract}
BACKGROUND AND PURPOSE: Imaging a cochlear implant with CT is challenging because of implant-induced artifacts, anatomic cochlear variations, and lack of standard terminology for cochlear anatomy. The purposes of this project were to determine whether the cochlear implant tip was more accurately located on oblique CT reformations than on standard images, to review radiology reports for accurate cochlear implant locations, and to assess agreement between an implant surgeon and neuroradiologist by using standardized cochlear anatomy terminology for cochlear implant depth.
\end{abstract}

MATERIALS AND METHODS: In this retrospective study, a neuroradiologist and an implant surgeon independently viewed temporal bone CT images of 36 ears with cochlear implants. Direct axial images, standard coronal reformations, and oblique reformations parallel to the cochlea were compared to determine implant tip location, which was described by using a proposed standardized quadrant terminology. Implant locations were compared with the initial formal report generated by the original interpreting neuroradiologist.

RESULTS: Thirty-six temporal bones with cochlear implants underwent CT interpretation for implant location. Interobserver agreement was similar when comparing cochlear implant tip location by using a quadrant nomenclature on axial and coronal images and on oblique reformations. Clinical radiology reports all were imprecise and ambiguous in describing the location of the cochlear implant tip.

CONCLUSIONS: Accurate determination of insertion depth of the cochlear implant array can be determined by assessment of the implant tip on axial, coronal, and oblique CT images, but description of the tip location can be inaccurate due to lack of standardized terminology. We propose using a standardized terminology to communicate tip location by using the round window as the zero reference and quadrant numbering to describe cochlear turns. This results in improvement in radiology report accuracy and consistency regarding the cochlear implant insertion depth.

ABBREVIATION: $\mathrm{Cl}=$ cochlear implant

maging of the cochlea is difficult due to its small size, oblique axis, and anatomic variations within the temporal bone. When a cochlear implant (CI) is in place, CT can be especially difficult because of artifacts from the implant. Furthermore, the absence of standard cochlear turn nomenclature renders radiology reports

Received September 23, 2013; accepted after revision July 30, 2014.

From the Department of Otolaryngology and Head and Neck Surgery (C.C.C.), University of Minnesota, Minneapolis, Minnesota; Department of Otolaryngology and Head and Neck Surgery (N.W.T.), and Head and Neck Imaging Section (P.A.H.), Department of Radiology and Imaging Sciences, Emory University School of Medicine, Atlanta, Georgia; and Division of Neuroradiology (H.R.H.), Department of Radiology, University of Utah School of Medicine, Salt Lake City, Utah.

Preliminary data previously presented as a poster at: Annual Meeting of the Triologic Society at Combined Otolaryngology Spring Meetings, April 28-30, 2011; Chicago, Illinois.

Please address correspondence to Patricia A. Hudgins, MD, Department of Radiology and Imaging Sciences, Emory University Hospital, 1364 Clifton Rd, NE, Atlanta, GA 30322; e-mail: phudgin@emory.edu

http://dx.doi.org/10.3174/ajnr.A4105 regarding CI insertion depth difficult to understand. Anatomic descriptions of the cochlea vary throughout the basic science, radiology, pathology, and otolaryngology literature. Otolaryngology textbooks typically describe a basal turn, medial turn, and apical turn, but cochlear turn language is not standardized across all texts. Occasionally angle degrees, assuming $360^{\circ}$ in a circle, are used as a descriptor for the multiple turns, with the round window niche as the zero-degree reference. ${ }^{1}$

It is important to be able to describe the CI tip location because electrode-array insertion depth and location are relevant factors when considering hearing and speech outcomes following CI insertion. ${ }^{1-4}$ Audiologic outcomes are better when the electrode array is inserted into the scala tympani, allowing better stimulation of the spiral ganglion neural elements. Multidetector CT can detect the scala tympani in cadaveric specimens, ${ }^{5}$ but application to routine clinical postimplantation imaging is limited. Multiple imaging methods have been used to determine CI electrode depth 
postoperatively, including conventional radiology, ${ }^{4}$ fluoroscopy, ${ }^{3}$ conebeam CT, ${ }^{6}$ fusion of conventional radiography and CT images, ${ }^{7}$ and multidetector CT. ${ }^{5,8}$ Lecerf et al ${ }^{9}$ showed that midmodiolar reconstructed CT on cadaver temporal bones can be used as an effective method for neuroradiologists to assess the location of CIs in either the scala vestibuli or scala tympani with high sensitivity and specificity. Ideally, the radiology report would routinely include objective measures of the CI tip insertion depth and a scalar chamber assignment so that these can be compared against the clinically desired position of the electrode tip.

In 2010, a consensus panel comprising radiologists, otologists, and researchers developed a cochlear coordinate system ${ }^{10}$ to have an objective method for comparing study and research outcomes by using a standard description of the cochlea. The consensus terminology developed for describing cochlear anatomy uses the round window as the zero-degree point. ${ }^{11}$ This $3 \mathrm{D}$ cylindric coordinate system uses the basal turn of the cochlea as the $\mathrm{x}$ and $\mathrm{y}$ planes and the center of the modiolus as the z-axis, with the zerodegree point described as the round window.

In our experience, this standard description of cochlear anatomy is not routinely used in the radiology report and, in fact, has not been emphasized in the radiology scientific literature. The lack of standard terminology when describing cochlear anatomy makes it nearly impossible to know from reading a radiology report how far the electrode array extends into the cochlea. Current clinical reports from radiologists often ambiguously describe how far the electrode array extends into the cochlea, and they lack consistent descriptions of the cochlea and implant array from one radiologist to the next.

We had 3 goals: first, to determine whether the CI tip was more accurately determined on direct axial images, standard coronal reformations, or oblique CT reformations obtained parallel to the basal portion of the first cochlear turn; second, to review the formal CT interpretations to see whether the CI tip location was accurately reported; and third, to assess agreement between an implant surgeon and neuroradiologist when asked to identify the CI-array depth by using the 2010 consensus terminology.

\section{MATERIALS AND METHODS}

This institutional review board-approved retrospective study included children younger than 18 years of age with CIs who underwent CT of the temporal bones during a 5-year period. Thirty-six temporal bones (33 children) with CIs were identified. Eighteen of the patients were male; 15 were female. The average age at the time of CT imaging was 8.4 years (range, 16 months to 16 years). The imaging was performed, on average, 3 years following implantation, with a range of 11 months to 11 years. Most of the implants were Cochlear Corporation devices (Englewood, Colorado) (25/36), with the remaining being MED-EL (Durham, North Carolina) (6/36) and Advanced Bionics (Valencia, California) (5/36) devices.

All patients included had undergone cochlear implantation for bilateral sensorineural hearing loss and were included regardless of cochlear anatomy. All CIs had been placed through a standard posterior tympanotomy, either through the round window or within millimeters of the round window. All had complete insertion achieved. The children underwent CT of the temporal bone due to concern for implant function or in preparation for

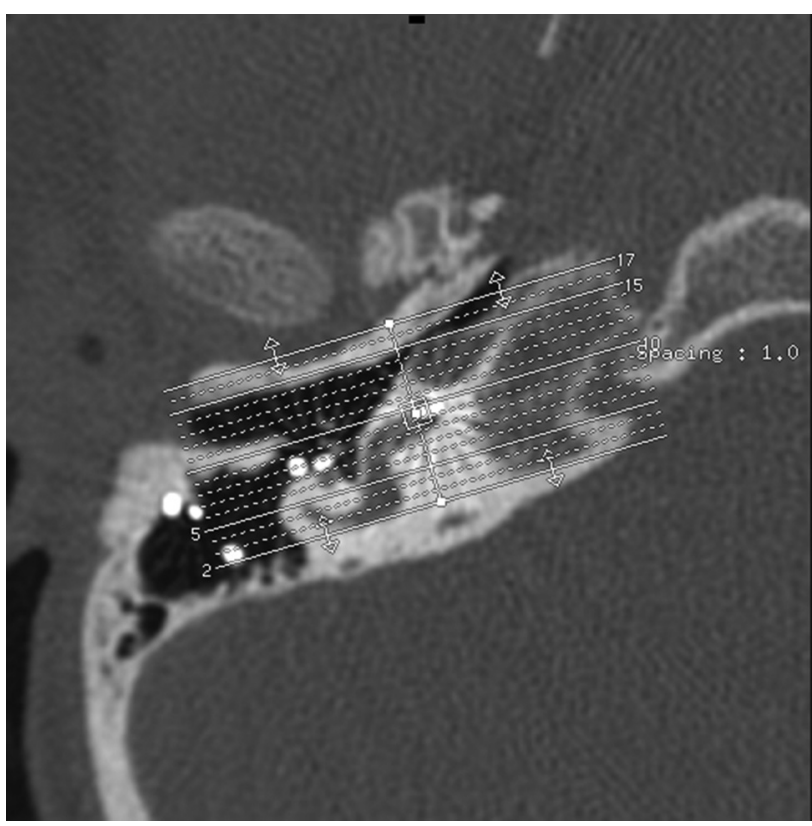

FIG 1. Oblique reformations along the plane of quadrant I. Axial CT, right temporal bone, through the first cochlear turn, quadrant I, showing the slab thickness for oblique reformations.

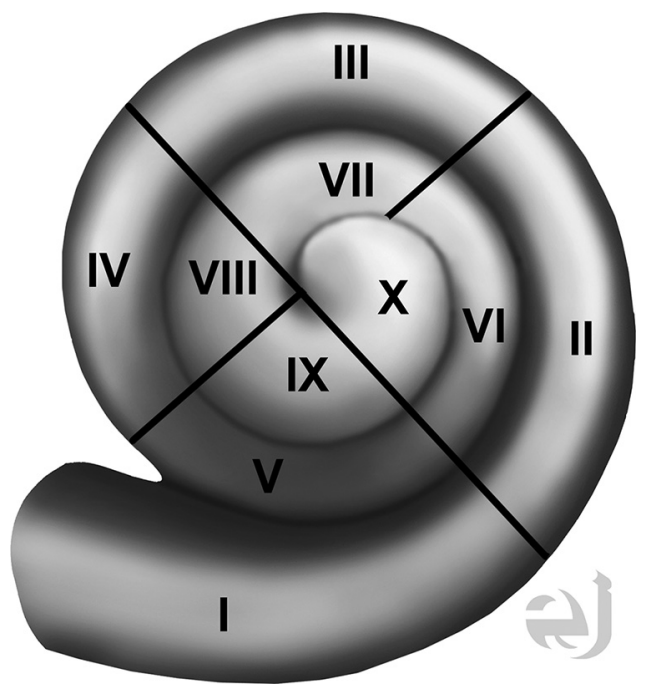

FIG 2. Drawing of the cochlea with quadrants numbered. Drawing of all cochlear turns, with the quadrants numbered I-X. Use of this approach in reporting the $\mathrm{Cl}$ tip will standardize discussion of implant tip location. Courtesy of Eric Jablonowski, medical illustrator.

contralateral implantation. Images were obtained without intravenous contrast in the axial plane from immediately beneath the mastoid tip to the top of the petrous apex $(110 \mathrm{kV}[$ peak]; $120 \mathrm{~mA}$; FOV, $17-18 \mathrm{~cm} ; 0.625-\mathrm{mm}$ section thickness). Axial source images were used to reconstruct coronal and oblique (along the plane of the basal portion of the first turn of the cochlea, Fig 1) CT images. If multiple scans were obtained in the study period, only the most recent imaging was used for study purposes. Exclusion criteria were imaging not performed at our tertiary care facility and images with motion artifacts precluding adequate reformations.

A neuroradiologist and an implant surgeon independently reviewed 3 sets of images: the axial images, coronal reformations, and oblique reformations along the plane of the basal portion of 
the first cochlear turn. All images were interpreted on a PACS. Coronal reformations perpendicular to the axial images were performed by the CT technologist and were submitted directly to the PACS. The oblique reformations were created by the implant surgeon and the neuroradiologist, by using a postprocessing function of the workstation. Both readers were blinded to implant function and the implant tip location regarding the depth of the implant as described on surgical notes. The location of the implant position within the cochlea was described by using an approach based on consensus terminology for standard terms regarding cochlear anatomy. ${ }^{10}$ Results were recorded by using a 4-quadrant system for each $360^{\circ}$ turn of the cochlea (noted as numerals I-X), with the round window as a zero-degree reference point (Fig 2). For exam-

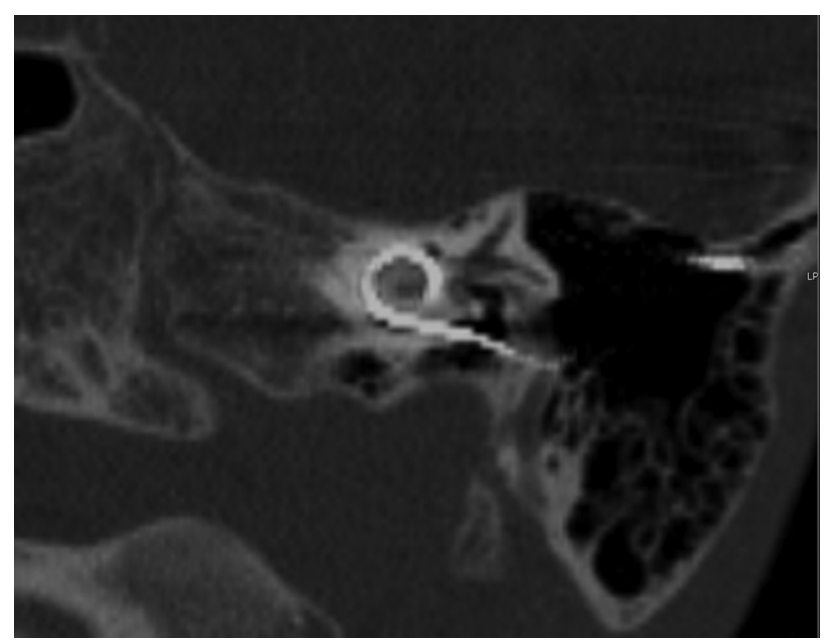

FIG 3. A 7-year-old boy with a left-sided cochlear implant. Single oblique reformation shows the $\mathrm{Cl}$ tip in quadrant IV.

Table 1: Agreement about quadrant of cochlear implant depth in 36 ears ( 33 patients)

\begin{tabular}{cccccc}
\hline & \multicolumn{5}{c}{ Expression of Agreement } \\
\cline { 2 - 6 } Agreement of Concern & Spearman $r$ & $\begin{array}{c}\text { Same } \\
\text { Quadrant }\end{array}$ & $\begin{array}{c}\text { 1-Quadrant } \\
\text { Difference }\end{array}$ & $\begin{array}{c}\text { 2-Quadrant } \\
\text { Difference }\end{array}$ & $\begin{array}{c}\text { 3-Quadrant } \\
\text { Difference }\end{array}$ \\
\hline $\begin{array}{c}\text { Rdr 1: axial-coronal vs } \\
\text { reformatted images }\end{array}$ & $.90, P<.001$ & 21 & 13 & 2 & 0 \\
$\begin{array}{c}\text { Rdr 2: axial-coronal vs } \\
\text { reformatted images }\end{array}$ & $.89, P<.001$ & 20 & 15 & 1 & 0 \\
$\begin{array}{c}\text { Rdr 1 vs 2: axial-coronal } \\
\text { images }\end{array}$ & $.88, P<.001$ & 16 & 18 & 2 & 0 \\
$\begin{array}{c}\text { Rdr 1 vs 2: reformatted } \\
\text { images }\end{array}$ & $.90, P<.001$ & 16 & 16 & 4 & 0 \\
\hline
\end{tabular}

Note:-Rdr indicates reader.

Table 2: Examples from dictations by neuroradiologists interpreting temporal bone CT obtained for CI position

Examples
"Enters the cochlea at the basal turn via the cochlear promontory and extends to the apical
half turn."
"Left cochlear implant in place."
"Implant lead then enters the round window, passes into the cochlea and terminates near
the apical half turn."
"Electrode intact and normal in course."
"Electrode continues to the cochlea where it has appropriate turns."
"Enters the basal turn of the cochlea, looping within the cochlea, and terminating near the
apex."
"It extends to, but does not go through, the aperture."
"Through the round window and filling the basal and middle turns of the cochlea."

ple, insertion into only the inferior quadrant of the first turn would be termed "quadrant I," insertion into the inferior quadrant of the second turn would be termed "quadrant V," and insertion into the posterior quadrant of the second turn would be termed "quadrant VIII" insertion. The data obtained were then compared with the original report that had been generated at the time of imaging by a neuroradiologist with a Certificate of Added Qualification. Interobserver agreement between the implant surgeon and neuroradiologist was determined for axial and coronal images, and for oblique reformations within the plane of the basal turn of the cochlea.

A consensus approach was used to resolve any cases in which the reading of the implant tip differed by $>2$ quadrants.

Statistical Analysis. Interobserver agreement was determined by using the Spearman rank correlation coefficient (Spearman $r$ ).

\section{RESULTS}

Interobserver agreement was excellent for the 3 planes assessed: axial, coronal, and oblique reformations (Fig 3). Interobserver agreements by expression of agreement among quadrants (Spearman $r$ ) are shown in Table 1. The implant surgeon's and neuroradiologist's readings did not differ by $>2$ quadrants in any given patient. Readings that differed were usually because the implant tip was near the interface between 2 quadrants.

Radiology reports for each CT study, written before the new consensus terminology, ambiguously described how far the electrode array extended into the cochlea. Examples of the description of the CI array from radiology reports are demonstrated in Table 2.

\section{DISCUSSION}

Using consensus terminology, we sought to evaluate agreement between an implant surgeon and neuroradiologist when asked to identify CI insertion depth. Interobserver agreement was excellent for direct axial and reformatted coronal planes and oblique reformations in the plane of the basal portion (quadrant I) of the first cochlear turn. Although CI tip location could be determined by using the axial and coronal planes, the oblique images reformatted in the plane of the basal portion of the first cochlear turn helped to confirm tip location, usually on a single image. Both the otologic surgeon and the neuroradiologist subjectively thought that the implant tip was easier and faster to determine on the oblique reformations, though this opinion was not objectively measured. We believe the use of standardized terminology allowed the observers to more accurately describe the location of the implant array and resulted in less uncertainty when describing cochlear anatomy.

Clinical radiology reports written without using consensus terminology have ambiguously described how far the 
electrode array extended into the cochlea (Table 2), making it nearly impossible for the reader to consistently determine the exact location of the CI insertion depth. The electrodes on the CI can often be seen and even counted on the CT scan, but it may be difficult to see each electrode, depending on the manufacturer. The CI tip, however, can always be appreciated on CT. The complex anatomy of the cochlea and individual differences in size, form, and location within the temporal bone often add to the level of difficulty in accurately describing the location of the electrode array. Most implant surgeons probably review the CT scans themselves, but a more accurate written interpretation in the medical record is obviously necessary.

Factors that affect CI function include both patient and implant variables. For example, the duration of hearing loss and the age of implantation are patient-related factors that affect CI outcomes. ${ }^{12}$ While early research suggested that the depth of insertion of the CI was an important variable affecting function, more recent data show that consideration of depth alone is an oversimplification of assessing CI function. In fact, one article has shown poorer function as the depth of insertion increases. ${ }^{2}$ Implant function may be affected by whether the implant is in the scala tympani, as opposed to the scala vestibuli or if it hugs the modiolus, as opposed to being lateral in the cochlea. High-resolution CT has been able to predict the scala location of the implant. ${ }^{5,9}$ Although this has been described in postmortem temporal bones on CT, it is possible that the scalar location will be routinely determined in vivo. Therefore, as understanding of variables affecting CI function progresses, imaging interpretation will likely go beyond simply describing the location of the implant tip.

Use of the consensus terminology enables multiple experienced viewers to communicate implant-array location with less ambiguity than previous methods. ${ }^{10}$ Throughout the literature, especially when comparing otolaryngologic and radiologic textbooks, descriptions of cochlear anatomy vary significantly. This variation is especially seen when the cochlea is malformed, without the usual number of turns. Our population did not include severely malformed cochleas, and the quadrant nomenclature could not be used in severely malformed cochleas because it would not be directly applicable. Along with consensus terminology, we have found that easier determination of the insertion depth of CI arrays and more effective description of insertion depth are enabled by cochlea-specific reformation planes set in the plane of the cochlea and by using the round window niche as the zero reference. As implant technology progresses, the neuroradiologist may be asked to be more precise about implant location in the cochlea, and a quadrant approach to describe implant tip location can serve as the basis for those interpretations.

\section{CONCLUSIONS}

CT reformations parallel to the cochlear turns and use of standardized terminology regarding cochlear anatomy facilitated consistent reporting of the depth of the CI insertion. We propose universal use of standard terminology by using a numeric quadrant approach instead of naming individual cochlear turns. Until such a system of standard terminology for reporting CI insertion depth is adopted, comparisons regarding insertion depth and clinical outcomes among various research articles will remain problematic.

\section{ACKNOWLEDGMENTS}

The authors wish to acknowledge Eric Jablonowski, artist.

Disclosures: Patricia A. Hudgins-UNRELATED: Consultancy: Amirsys, Comments: medical education company (I am a consultant and author); Royalties: Amirsys, Comments: royalties for medical education company publishing; Ownership Interest: Amirsys. H. Ric Harnsberger-UNRELATED: Ownership Interest: CEO, Amirsys. Norman W. Todd-UNRELATED: Consultancy: National Institutes of Health, Comments: Data Safety Monitoring Board member for study of Auditory-Verbal teletherapy; Grants/Grants Pending: MED-EL, ${ }^{*}$ Comments: clinical study about the potential usefulness of electrically elicited stapedius reflexes in cochlear implant recipients; Patents (planned, pending or issued): Force Threshold Gauge, ${ }^{*}$ Comments: Patent application submitted. *Money paid to the institution.

\section{REFERENCES}

1. Verbist BM, Ferrarini L, Briaire JJ, et al. Anatomic considerations of cochlear morphology and its implications for insertion trauma in cochlear implant surgery. Otol Neurotol 2009;30:471-77

2. Finley CC, Holden TA, Holden LK, et al. Role of electrode placement as a contributor to variability in cochlear implant outcomes. Otol Neurotol 2008;29:920-28

3. Roland JT Jr, Fishman AJ, Alexiades G, et al. Electrode to modiolus proximity: a fluoroscopic and histologic analysis. Am J Otol 2000; 21:218-25

4. Xu J, Xu SA, Cohen LT, et al. Cochlear view: postoperative radiography for cochlear implantation. Am J Otol 2000;21:49-56

5. Lane JI, Driscoll CL, Witte RJ, et al. Scalar localization of the electrode array after cochlear implantation: a cadaveric validation study comparing 64-slice multidetector computed tomography with microcomputed tomography. Otol Neurotol 2007;28:191-94

6. Schuman TA, Noble JH, Wright CG, et al. Anatomic verification of a novel method for precise intrascalar localization of cochlear implant electrodes in adult temporal bones using clinically available computed tomography. Laryngoscope 2010;120:2277-83

7. Whiting BR, Bae KT, Skinner MW. Cochlear implants: three-dimensional localization by means of coregistration of CT and conventional radiographs. Radiology 2001;221:543-49

8. Verbist BM, Joemai RM, Teeuwisse WM, et al. Evaluation of 4 multisection CT systems in postoperative imaging of a cochlear implant: a human cadaver and phantom study. AJNR Am J Neurorad 2008;29:1382-88

9. Lecerf $\mathrm{P}$, Bakhos D, Cottier JP, et al. Midmodiolar reconstruction as a valuable tool to determine the exact position of the cochlear implant electrode array. Otol Neurotol 2011;32:1075-81

10. Verbist BM, Skinner MW, Cohen LT, et al. Consensus panel on a cochlear coordinate system applicable in histologic, physiologic, and radiologic studies of the human cochlea. Otol Neurotol 2010; 31:722-30

11. Verbist BM, Joemai RM, Briaire JJ, et al. Cochlear coordinates in regard to cochlear implantation: a clinically individually applicable 3 dimensional CT-based method. Otol Neurotol 2010;31:738-44

12. Holden LK, Finley CC, Firszt JB, et al. Factors affecting open-set word recognition in adults with cochlear implants. Ear Hear 2013; $34: 342-60$ 